SHEP 01-21; OUTP 0146P

\title{
Fermion Masses and Mixing Angles from $S U(3)$ Family Symmetry
}

\author{
S. F. King ${ }^{\dagger}$ and G. G. Ross ${ }^{\ddagger}$ \\ †Department of Physics and Astronomy, University of Southampton, \\ Southampton, SO17 1BJ, U.K. \\ †Department of Physics, Theoretical Physics, University of Oxford, \\ 1 Keble Road, Oxford OX1 3NP, U.K.
}

\begin{abstract}
We propose a model based on $S U(3)$ family symmetry which leads to a successful description of quark and lepton masses and mixing angles including approximate bi-maximal mixing in the neutrino sector suitable for the LOW or quasi-vacuum solar solutions, with the atmospheric angle predicted to be accurately maximal due to the $S U(3)$ symmetry. The model predicts a $\mathrm{CHOOZ}$ angle $\theta_{13} \sim\left|V_{u b}\right|$. The $S U(3)$ symmetry can also ensure the near degeneracy of squarks and sleptons needed to avoid large flavour changing neutral currents.
\end{abstract}




\section{Introduction}

The origin of fermion masses and mixing angles has been a long standing puzzle[1]]. Our determination of the mass matrices has been improved by new experimental information on both the quark and lepton sectors. Particularly intriguing is the appearance of an almost maximal neutrino mixing angle $\theta_{23}=45^{0}$ responsible for atmospheric neutrino oscillation and the suggestion of a near maximal mixing angle $\theta_{12} \approx 45^{0}$ in order to describe solar neutrino oscillation [2]. Apart from the CHOOZ angle this contrasts sharply with the quark mixing angles which are all small.

In this paper we shall show that a near maximal mixing angle may be a signal of an underlying non-Abelian family symmetry and demonstrate how vacuum alignment in such a model can lead to maximal mixing. Further we argue that the quark mass matrix is also indicative of a nonAbelian symmetry, although in this case the symmetry is responsible for the smallness of quark mixing angles. The origin of this apparent contradiction is the see-saw mechanism which alters the form of the neutrino mass matrix from that of the quarks and charged leptons.

Non-Abelian family symmetries have been considered before 3. In supersymmetric theories they offer an elegant solution to the flavour problem, ensuring that the squarks are nearly degenerate and thus suppressing flavour changing neutral currents (FCNC). Given that the third family of quarks and leptons is much heavier, and that the dominant contribution to FCNC comes from the light generations, early attempts to develop such theories considered the non-Abelian group $S U(2)$ acting on the first two generations only[4. However these theories do not offer an easy explanation for the near maximal neutrino mixing in the neutrino sector involving the second and third generations. Such mixing requires a correlation between the $(2,2)$ and $(2,3)$ elements of the mass matrix. This strongly suggests an underlying non-Abelian symmetry involving the third generation too. For this reason we consider here a $S U(3)$ family symmetry acting on all three generations with the view to addressing both these questions. A gauged $S U(3)$ family symmetry which could provide a dynamical origin for the three observed families of quarks and leptons has a long history in the literature [5]. However as far as we are aware previous $S U(3)$ models have not been able to use the symmetry to relate mass matrix elements after spontaneous symmetry breaking. Here we show how this can be done via a novel mechanism for vacuum alignment.

In this paper we shall consider a particular class of $S U(3)$ model which gives a successful description of quark masses and mixing angles, and simultaneously gives approximately bimaximal leptonic mixing with $\theta_{23}$ fixed to be almost maximal by a combination of $S U(3)$ and vacuum alignment. In both cases the origin of the mixing angles is controlled by the non-Abelian vacuum structure relating the second and third generations

It is instructive to see how data favours such a structure in the quark mass matrix. A recent analysis of all experimental measurements relating to quark masses and mixings including the latest measurements at BaBar and BELLE has found the following form for the up and down quark mass matrices [7]

$$
\frac{M^{u}}{m_{t}}=\left(\begin{array}{ccc}
0 & b^{\prime} \epsilon^{3} & c^{\prime} \epsilon^{3} \\
b^{\prime} \epsilon^{3} e^{i \phi^{\prime}} & \epsilon^{2} & a^{\prime} \epsilon^{2} \\
? & ? & 1
\end{array}\right)
$$

\footnotetext{
${ }^{1}$ With an Abelian $U(1)$ family symmetry [6] the mixing angle $\theta_{23}$ cannot be enforced to be accurately maximal, but it may be large.
} 
and

$$
\frac{M^{d}}{m_{b}}=\left(\begin{array}{ccc}
0 & b \bar{\epsilon}^{3} & c \bar{\epsilon}^{3} \\
b \bar{\epsilon}^{3} e^{i \phi} & \bar{\epsilon}^{2} & a \bar{\epsilon}^{2} \\
? & ? & 1
\end{array}\right)
$$

The equality of the magnitudes of the $(1,2)$ and $(2,1)$ elements leads to the successful Gatto, Sartori, Tonin (GST) relation 10 for a $(1,1)$ texture zero. The $(3,1)$ and $(3,2)$ elements are only weakly constrained because measurement of the quark masses and the CKM matrix does not provide enough information to determine the full quark mass matrices. The parameters of the up quark mass matrix are given by $\epsilon=0.05, b^{\prime} \simeq 1$ while $a^{\prime}$ and $c^{\prime}$ are very weakly constrained. The parameters of the down quark mass matrix are much better determined with

$$
\begin{aligned}
\bar{\epsilon} & =0.15 \pm 0.01, \quad b=1.5 \pm 0.1, \quad a=1.31 \pm 0.14 \\
|c| & =0.4 \pm 0.02, \quad \psi=24^{0} \pm 3^{0} \text { or } \\
|c| & =1.27 \pm 0.05, \quad \psi=-58^{0} \pm 5^{0}
\end{aligned}
$$

where $c=|c| e^{i \psi}$. The fact that the $(2,2)$ and $(2,3)$ matrix elements are very similar in magnitude is required by the smallness of $V_{c b}$. It suggests a relation between these elements, suggestive of a non-Abelian symmetry. The recent data now also requires that the $(1,2)$ and $(1,3)$ are also quite similar, supporting this interpretation and disfavouring the promising symmetric texture zero structure with zeroes in the $(1,1)$ and $(1,3)$ elements 8 , 넬.

In the present paper we shall propose a model of fermion masses and mixing angles based on an $S U(3)$ family symmetry, together with certain discrete symmetries which are required to forbid unwanted operators. The model we propose will give rise to Yukawa matrices of the form

$$
Y \approx\left(\begin{array}{ccc}
O\left(\epsilon^{8}\right) & \lambda \epsilon^{3} & \lambda \epsilon^{3} \\
-\lambda \epsilon^{3} & \epsilon^{2} & \epsilon^{2} \\
-\lambda \epsilon^{3} & \epsilon^{2} & 1
\end{array}\right)
$$

in leading order of the expansion parameter, $\epsilon$. The coupling $\lambda$ is not determined by the symmetry but is expected to be of $O(1)$. The matrix has a hybrid symmetry, being symmetric in the lower block $(3,2)=(2,3)$ and antisymmetric in the remaining entries $(2,1)=-(1,2),(3,1)=-(1,3)$. This form applies to the up and down quarks, to the charged leptons and to he neutrino Dirac couplings between left- and right- handed neutrino components. In general there is an independent expansion parameter $\epsilon$ for each Yukawa matrix. Note that at leading order each Yukawa is described by only two free parameters! The right- handed Majorana coupling matrix has a different structure.

\section{The $S U(3)$ family symmetry.}

Here we outline the structure of the model, postponing details to later sections. We start with an $S U(3)$ family symmetry which commutes with the Standard Model (SM) gauge group. We

\footnotetext{
${ }^{2}$ If one allows for an asymmetric form of the quark mass matrices with large entries below the diagonal it is still possible to have a $(1,3)$ zero[] . Here we concentrate on symmetric structures which naturally accommodate the successful GST relation.

${ }^{3}$ Such matrices have been considered before, eg see [9], but not with a symmetry ensuring equality of matrix elements.
} 
shall construct a supersymmetric version of the theory so, apart from the modifications needed to implement the family symmetry, we have the basic structure of the minimal supersymmetric SM (MSSM) in which fermions belong to chiral supermultiplets. For simplicity of presentation, we will treat the supersymmetric structure as implicit. The family symmetry assignments for the left-handed quarks and leptons are:

$$
\psi_{i} \in\left(Q_{i}, L_{i}\right) \sim 3, \quad \psi_{i}^{c} \in\left(U_{i}^{c}, D_{i}^{c}, E_{i}^{c}, N_{i}^{c}\right) \sim 3
$$

where $i=1,2,3$ are $S U(3)$ labels. To build a viable model we need spontaneous breaking

$$
S U(3) \longrightarrow S U(2) \longrightarrow \text { Nothing }
$$

Note that whenever we write $S U(3)$ it will always refer to the new family symmetry and $S U(2)$ will always refer to its subgroup. These should not be confused with the SM gauge group factors with which the family symmetry commutes.

The Higgs fields responsible for the above symmetry breaking are the $S U(3)$ antitriplets (but SM singlets) $\phi_{3}^{i}, \phi_{23}^{i}$, which develop vacuum expectation values (VEVs)

$$
<\phi_{3}>=\left(\begin{array}{c}
0 \\
0 \\
a_{3}
\end{array}\right), \quad<\phi_{23}>=\left(\begin{array}{l}
0 \\
b \\
b
\end{array}\right) \text {. }
$$

Note that $<\phi_{3}>$ can always be rotated into the third position using $S U(3)$. The alignment of the $<\phi_{23}>$ VEVs is non-trivial and is the subject of a later section.

The leading contributions to the Yukawa matrices arise from operators which are quadratic in $\phi_{3}^{i}$ and $\phi_{23}^{i}$

$$
\left(\frac{1}{M^{2}} \psi_{i} \phi_{23}^{i} \psi_{j}^{c} \phi_{23}^{j}+\frac{1}{M_{3}^{2}} \psi_{i} \phi_{3}^{i} \psi_{j}^{c} \phi_{3}^{j}\right) H_{\alpha}
$$

where $H_{\alpha}$ are the two Higgs doublets of the MSSM, which are $S U(3)$ singlets, but carry the usual electroweak quantum numbers. If $\psi^{c}=U^{c}, N^{c}$ then $\alpha=2$, while if $\psi^{c}=D^{c}, E^{c}$ then $\alpha=1,2$. The difference between the mass scales $M$ and $M_{3}$ is discussed below.

\subsection{The operator mass scale}

The origin of these operators lies in the physics beyond the SM at some higher energy scale and the inverse mass scale associated with these operators reflects this higher scale. For example the operator may be due to a Higgs "messenger" sector through mixing of the MSSM Higgs with heavy vectorlike Higgs fields 11]. In this case the inverse mass scale is that of the heavy Higgs. Since the heavy Higgs carrying the weak hypercharge of the $H_{\alpha}$ in the $\alpha=1,2$ sectors may be different, the case when $\psi^{c}=U^{c}, N^{c}$ is expected to have a different inverse mass scale, $M=M^{u}$, from the case when $\psi^{c}=D^{c}, E^{c}$ for which $M=M^{d}$. The operators may also arise through mixing of the quarks with heavy vectorlike quarks but again we expect the associated operator mass scale to differ for the up quark and down quark sectors. When $\phi_{23}$ is replaced by its $\mathrm{VEV}$, the operators of eq(9) generate equal $(2,2)=(2,3)=(3,2)$ elements of $O\left(b^{2} / M^{2}\right)$ in the 23 block of Eq.5. As we shall see later this equality is responsible for the maximal neutrino mixing angle $\theta_{23}$. Due to the different inverse mass scale the expansion parameters in the up 
and the down sectors are expected to be different. We shall denote the expansion parameter in the up sector by $\epsilon \equiv b / M^{u}$ and that in the down sector by $\bar{\epsilon} \equiv b / M^{d}$.

Some comment about the fact that the leading term in Eq.5 for both the up and the down sectors is of $O(1)$ is in order. At first sight it would seem that, because the $(3,3)$ term comes from a higher dimension operator, its magnitude should differ in the up and down sectors for the same reason that the $O\left(\epsilon^{2}, \bar{\epsilon}^{2}\right)$ terms differ. However if $a_{3}>M$, where $M$ is the generic mass scale associated with the up or the down sector, the expansion parameter is not $a_{3} / M$ but rather $a_{3} / M_{3}$ where $M_{3} \approx \sqrt{M^{2}+a_{3}^{2}}$. The reason for this is that the heavy messenger field responsible for generating the higher dimension operator necessarily has a contribution to its mass coming from $a_{3}$ since it couples to $\phi_{3}$. In addition it will have other contributions characterised by $M$. Obviously the largest term dominates giving the result above. Here we assume that $a_{3} \geq M>b$ so that $\phi_{3}$ provides the first stage of breaking of $S U(3)$, while the second stage, triggered by the $\mathrm{VEV}$ of $\phi_{2}$, is below the mass scale of the heavy sector and so generates small effects characterised by the expansion parameters, $\epsilon$ and $\bar{\epsilon}$. In what follows we shall often refer to the expansion parameter as $\epsilon$ but it should be remembered that this will be $\bar{\epsilon}$ in the down quark sector. In this paper we shall not consider the details of the heavy sector responsible for the generation of the operators but content ourselves with the construction of the effective low energy theory, including operators consistent with the symmetries of the theory.

\subsection{Subleading operators}

The operators which are mixed in $\phi_{3}^{i}, \phi_{23}^{i}$ give the order $\epsilon^{3}$ contributions to the $(2,3),(3,2),(3,3)$ elements in Eq.5, and so we require them to be suppressed by $\epsilon^{2}$ relative to the quadratic operators in Eq.9,

$$
\frac{\epsilon^{2}}{M M_{3}}\left(\psi_{i} \phi_{23}^{i} \psi_{j}^{c} \phi_{3}^{j}+\psi_{i} \phi_{3}^{i} \psi_{j}^{c} \phi_{23}^{j}\right) H_{\alpha}
$$

If the mixed operators were not suppressed then they would imply that the $(2,3)$ element is of $O(\epsilon)$, larger than the $(2,2)$ element of $O\left(\epsilon^{2}\right)$, and would lead to the bad relation $\left|V_{c b}\right| \simeq$ $\sqrt{m_{s} / m_{b}}$. Here we require a further $O\left(\epsilon^{2}\right)$ suppression for reasons that will become apparent. In order to achieve this suppression we introduce a discrete symmetry $Z_{2}$ under which $\phi_{3}$ and $\phi_{23}$ have opposite parity. This allows the quadratic operators, but forbids the leading order mixed operators. The latter are only allowed at higher order with the $\epsilon^{2}$ suppression factor.

In order to generate Yukawa entries in the first row and column, we need to introduce the $S U(3)$ triplet (SM singlet) Higgs $\bar{\phi}_{3, i}, \bar{\phi}_{23, i}$, which we shall show develops VEVs along $D-$ and $F$-flat directions.

$$
<\bar{\phi}_{3}>=\left(\begin{array}{c}
0 \\
0 \\
a_{3}
\end{array}\right), \quad<\bar{\phi}_{23}>=\left(\begin{array}{r}
0 \\
b \\
-b
\end{array}\right) .
$$

The operators responsible for the first row and column of the Yukawa matrices are then

$$
\begin{gathered}
\frac{\epsilon^{2}}{M}\left(\epsilon^{i j k} \psi_{i} \bar{\phi}_{23, j} \psi_{k}^{c}\right) H_{\alpha} \\
\frac{\epsilon^{6}}{M^{2} M_{3}^{2}}\left(\epsilon^{i j k} \psi_{i} \bar{\phi}_{3, j} \bar{\phi}_{23, k}\right)\left(\epsilon^{l m n} \psi_{l}^{c} \bar{\phi}_{3, m} \bar{\phi}_{23, n}\right) H_{\alpha}
\end{gathered}
$$


The inclusion of triplet Higgs in addition to the antitriplets introduced previously allows the antisymmetric tensor $\epsilon^{i j k}$ to be used to generate the first row and column of Eq.5. The operator in Eq.12 generates $(1,2)=(1,3)=-(2,1)=-(3,1)$ entries at order $\epsilon^{3}$, while the operator in Eq.13. generates the $(1,1)$ entry at order $\epsilon^{8}$. In the next section we discuss the origin of the required operator suppression factors.

\section{An $S U(3)$ Model}

\subsection{Operator Analysis}

In order to give a specific realisation of the basic scenario of the previous section we consider the full (SM singlet) scalar sector of the model summarised in Table 1. To ensure that the operators are only allowed at the specified orders we have introduced an R-symmetry under which all superpotential terms are required to have $R=2$. With these fields and VEVs introduced in the previous Section, the leading terms generating the fermion masses allowed by the family symmetries come from the superpotential

$$
\begin{aligned}
P & \left(\frac{1}{M_{3}^{2}} \psi_{i} \phi_{3}^{i} \psi_{j}^{c} \phi_{3}^{j}+\frac{1}{M^{2}} \psi_{i} \phi_{23}^{i} \psi_{j}^{c} \phi_{23}^{j}\right) H_{\alpha} \\
+ & \frac{1}{M_{3}^{3} M^{3}}\left(\psi_{i} \phi_{23}^{i} \psi_{j}^{c} \phi_{3}^{j}+\psi_{i} \phi_{3}^{i} \psi_{j}^{c} \phi_{23}^{j}\right) H_{\alpha}\left(\phi_{23}^{k} \bar{\phi}_{23, k}\right)\left(\phi_{3}^{l} \bar{\phi}_{3, l}\right) \\
+ & \frac{1}{M_{3}^{2} M^{3}}\left(\epsilon^{i j k} \psi_{i} \overline{\phi_{23}} \psi_{k}^{c}\right) H_{\alpha}\left(\phi_{3}^{l} \bar{\phi}_{23, l}\right)^{2} \\
+ & \frac{1}{M_{3}^{8} M^{8}}\left(\epsilon^{i j k} \psi_{i} \overline{\phi_{3 j}} \overline{\phi_{23}}\right)\left(\epsilon^{l m n} \psi_{l}^{c} \overline{\phi_{3 m}} \overline{\phi_{23 n}}\right) H_{\alpha}\left(\phi_{3}^{p} \bar{\phi}_{23, p}\right)^{6} \\
& +\frac{1}{M_{3}^{5} M^{4}}\left(\epsilon^{i j k} \psi_{i}^{c} \psi_{j} \bar{\phi}_{3, k}\left(\phi_{3}^{l} \overline{\phi_{23, l}}\right)^{4}+\epsilon^{i j k} \psi_{i}^{c} \overline{\phi_{3, j} \phi_{23, k}} \phi_{3}^{j} \psi_{j}\left(\phi_{3}^{l} \overline{\phi_{23, l}}\right)^{3}\right) H_{\alpha}
\end{aligned}
$$

where the terms in Eqs.14,17 correspond to the operators in Eqs.9, 10, 12, 13. They generate the correct suppression factors once the fields are replaced by their VEVs appropriately. Eq.(18) includes a further subleading term that will be important in the following discussion.

It is straightforward to show that the operators in $P$ are the leading ones allowed by the symmetry. In Table 2 we list the operators of leading dimension which would spoil the form of eq(5), together with the symmetry that forbids it. As we shall see in the next section, we may take the $\bar{\phi}_{2}$ VEV to be very small and so operators involving this field may be ignored. The remaining operators are forbidden by the family symmetries.

\subsection{Vacuum Alignment}

The critical feature of the model is the vacuum alignment which arranges for the fields $\phi_{23}, \bar{\phi}_{23}$ to acquire VEVs of equal magnitude in the 2 and 3 directions. Here we discuss how this comes about. The initial stage of symmetry breaking is triggered by VEVs for $\phi_{3}$ and $\overline{\phi_{2}}$. We assume that the fields have Yukawa couplings to the heavy sector fields which drive soft mass squared terms negative at some scale, $\Lambda$, through radiative corrections. The VEV cannot be larger than this scale as the effective potential has the form $m^{2}\left(\phi^{2}\right) \phi^{2}$ and clearly is positive above this scale 


\begin{tabular}{|ccccc|}
\hline Field & $\mathbf{S U}(\mathbf{3})$ & $\mathbf{Z}_{2}$ & $\mathbf{R}$ & $\mathbf{L}$ \\
\hline$\frac{\phi_{3}}{\phi_{3}}$ & $\overline{3}$ & + & +1 & 0 \\
$\frac{3}{\phi_{2}}$ & 3 & + & -2 & 0 \\
$\phi_{23}$ & $\overline{3}$ & - & 0 & 0 \\
$\phi_{23}$ & 3 & + & 0 & 0 \\
$\nu$ & $\overline{3}$ & + & +1 & 1 \\
$\bar{\nu}$ & 3 & + & +1 & -1 \\
$U$ & 1 & - & 1 & 0 \\
$X$ & 1 & + & +1 & 0 \\
$Y$ & 1 & + & +2 & 0 \\
$Z$ & 1 & - & 0 & 1 \\
\hline
\end{tabular}

Table 1: Transformation of the Higgs superfields under the family symmetries.

\begin{tabular}{|c|c|c|}
\hline Category & Forbidden Operator & Reason \\
\hline I & $\begin{array}{l}\psi_{i} \phi_{23}^{i} \psi_{j}^{c} \phi_{3}^{j} H_{\alpha} \\
\psi_{i} \phi_{23}^{i} \psi_{j}^{c} \phi_{3}^{j} H_{\alpha}\left(\bar{\phi}_{3 l} \phi_{3}^{l}\right) \\
\psi_{i} \phi_{23}^{i} \psi_{j}^{c} \phi_{3}^{j} H_{\alpha}\left(\bar{\phi}_{23 l} \phi_{3}^{l}\right) \\
\psi_{i} \phi_{23}^{i} \psi_{j}^{c} \phi_{3}^{j} H_{\alpha}\left(\bar{\phi}_{3 l} \phi_{23}^{l}\right)\end{array}$ & $\begin{array}{l}Z_{2} \\
Z_{2}, R \\
Z_{2}, R \\
R\end{array}$ \\
\hline II & $\begin{array}{l}\epsilon^{i j k} \psi_{i} \bar{\phi}_{23 j} \psi_{k}^{c} H_{\alpha} \\
\epsilon^{i j k} \bar{\psi}_{i} \bar{\phi}_{23 j} \psi_{k}^{c} H_{\alpha}\left(\bar{\phi}_{3 l} \phi_{3}^{l}\right) \\
\epsilon^{i j k} \psi_{i} \bar{\phi}_{23 j} \psi_{k}^{c} H_{\alpha}\left(\bar{\phi}_{23 l} \phi_{3}^{l}\right) \\
\epsilon^{i j k} \psi_{i} \bar{\phi}_{23 j} \psi_{k}^{c} H_{\alpha}\left(\bar{\phi}_{3 l} \phi_{23}^{l}\right)\end{array}$ & $\begin{array}{l}R \\
R \\
R \\
Z_{2}, R\end{array}$ \\
\hline III & $\begin{array}{l}\epsilon^{i j k} \psi_{i} \bar{\phi}_{3 j} \psi_{k}^{c} H_{\alpha} \\
\epsilon^{i j k} \psi_{i} \bar{\phi}_{3 j} \psi_{k}^{c} H_{\alpha}\left(\bar{\phi}_{3 l} \phi_{3}^{l}\right) \\
\epsilon^{i j k} \psi_{i} \bar{\phi}_{3 j} \psi_{k}^{c} H_{\alpha}\left(\bar{\phi}_{23 l} \phi_{3}^{l}\right) \\
\epsilon^{i j k} \psi_{i} \bar{\phi}_{3 j} \psi_{k}^{c} H_{\alpha}\left(\bar{\phi}_{3 l} \phi_{23}^{l}\right)\end{array}$ & $\begin{array}{l}R \\
R \\
R \\
Z_{2}, R\end{array}$ \\
\hline IV & $\begin{array}{l}\left(\epsilon^{i j k} \psi_{i} \bar{\phi}_{23 j} \bar{\phi}_{3 k}\right)\left(\psi_{l}^{c} \phi_{3}^{l}\right) H_{\alpha} \\
\left(\epsilon^{i j k} \psi_{i} \bar{\phi}_{23 j} \bar{\phi}_{3 k}\right)\left(\psi_{l}^{c} \phi_{23}^{l}\right)\left(\bar{\phi}_{3 m} \phi_{23}^{m}\right) H_{\alpha} \\
\left(\epsilon^{i j k} \psi_{i} \bar{\phi}_{23 j} \bar{\phi}_{3 k}\right)\left(\psi_{l}^{c} \phi_{23}^{l}\right) H_{\alpha}\end{array}$ & $\begin{array}{l}R \\
R \\
Z_{2}, R\end{array}$ \\
\hline V & $\left(\epsilon^{i j k} \psi_{i} \bar{\phi}_{23 j} \bar{\phi}_{3 k}\right)\left(\epsilon^{l m n} \psi_{l}^{c} \bar{\phi}_{23 m} \bar{\phi}_{3 n}\right) H_{\alpha}$ & $R$ \\
\hline
\end{tabular}

Table 2: Forbidden operators and the reason why they are excluded. 
and negative below. We suppose that such radiative effects trigger VEVs for $\phi_{3}$ and $\overline{\phi_{2}}$. Without loss of generality we can choose the basis such that $\phi_{3}^{T}=\left(\begin{array}{ccc}0 & 0 & a_{3}\end{array}\right)$. The alignment of the VEVs of $\phi_{3}$ and $\overline{\phi_{2}}$ is due to the term in the superpotential

$$
P_{1} \sim X \phi_{3} \overline{\phi_{2}}
$$

such that along the F-flat direction $\left|F_{X}\right|=0, \phi_{3}$ and $\overline{\phi_{2}}$ are orthogonal. Again without loss of generality we can choose the basis such that $\overline{\phi_{2}}=\left(\begin{array}{lll}0 & a_{2} & 0\end{array}\right)$. As we demonstrate in the Appendix, for a particular range of parameters, $D$ - flatness requires $\overline{\phi_{3}}=\left(\begin{array}{ccc}0 & 0 & a_{3}\end{array}\right)$.

Consider now the field $\phi_{23}$. We assume that due to different heavy sector interactions, its mass squared remains positive and its VEV is triggered by an F-term. The $S U(3)$ symmetry guarantees the equality of the components of the soft mass term $m_{23}^{2}\left|\phi_{23}\right|^{2}=m_{23}^{2}\left(\left|\phi_{23,1}\right|^{2}+\right.$ $\left.\left|\phi_{23,2}\right|^{2}+\left|\phi_{23,3}\right|^{2}\right)$. We will use this to obtain the vacuum alignment required. Consider the superpotential terms consistent with the symmetries of Table 1 .

$$
P_{2} \sim Y\left(\phi_{23} \overline{\phi_{2}} \phi_{23} \overline{\phi_{3}}-\mu^{4}\right), \quad P_{3} \sim U \phi_{23} \overline{\phi_{23}}
$$

Here $\mu$ is a mass scale associated with spontaneous symmetry breaking in the heavy sector. Requiring $\left|F_{Y}\right|=0$ implies $<\phi_{23,2} \phi_{23,3}>=\mu^{4} /\left(a_{2} a_{3}\right) \equiv b^{2}$ for $m_{23}^{2}>0$ 像. This is the vacuum alignment that will lead to maximal mixing in the neutrino sector. The resultant pattern of symmetry breaking along $m_{23}^{2} D$ - and $F$-flat directions, for the range of parameters detailed in the Appendix, is given in Table 3. Note that the fact the magnitude of the two VEVs in $\phi_{23}$, $\bar{\phi}_{23}$ are equal follows from the minimisation of $m_{23}^{2}\left|\phi_{23}\right|^{2}$ and that it is the underlying $S U(3)$ which ensures this by ensuring the soft mass terms are equal for all three components.

Finally we consider the fields $5, \bar{\nu}$ responsible for the Majorana masses. Below the scale of $S U(3)$ breaking, $a_{3}$, only the $S U(2)$ gauge bosons are light and contribute to the running of the $\widetilde{\nu}$ masses. Thus, below this scale, $\widetilde{\nu}_{1,2}$ become heavier than $\widetilde{\nu}_{3}$. However $\nu$ may also have Yukawa couplings to heavy states, which need not feel large $S U(3)$ breaking if they do not couple to $\phi_{3}$ in the superpotential. Such terms will drive $m_{\nu}^{2}$ negative in the usual way, driving a VEV in the lightest $\widetilde{\nu}_{3}$. Thus it is easy to align the VEV of $\nu$ without the addition of any superpotential "alignment" terms. However, if such terms are present they will skew the VEV from the direction favoured by the soft terms because such an F-term is not suppressed by the SUSY breaking scale that characterises the soft mass terms. When we come to consider neutrino masses it will be necessary to generate a component of the VEV along the $\nu_{1}$ direction. It is easy to construct just such a term via the introduction of an $F$-term consistent with the symmetries. Suppose we have the field $Z$ with quantum numbers shown in Table 3 . The allowed superpotential terms are

$$
P_{4} \sim Z \nu^{i}\left(\epsilon_{i j k} \phi_{23}^{j} \phi_{3}^{k}\left(\phi_{3} \bar{\phi}_{3}\right)+\bar{\phi}_{23 i}\left(\phi_{3} \bar{\phi}_{23}\right)^{2}\left(\bar{\phi}_{3} \phi_{23}\right)\right)
$$

Now the vanishing of $F_{Z}$ implies $i \nu^{1}-\epsilon_{\nu}^{2} \nu^{3}>=0$ so the VEV is skewed in the direction $j \nu>=$ $\left(\epsilon_{\nu}^{2}, 0,1\right) \sigma$.

\footnotetext{
${ }^{4}$ We take $b$ to be real, although our analysis is not sensitive to this assumption.

${ }^{5}$ These lepton number violating Higgs superfields should not be confused with neutrino superfields.
} 


\begin{tabular}{|c|c|}
\hline Field & VEV \\
\hline$\phi_{3}^{T}$ & $\left(\begin{array}{lll}0 & 0 & a_{3}\end{array}\right)$ \\
\hline$\overline{\phi_{3}}$ & $\left(\begin{array}{lll}0 & 0 & a_{3}\end{array}\right)$ \\
\hline$\overline{\phi_{2}}$ & $\left(\begin{array}{lll}0 & a_{2} & 0\end{array}\right)$ \\
\hline$\phi_{23}^{T}$ & $\left(\begin{array}{lll}0 & b & b\end{array}\right)^{\prime}$ \\
\hline$\overline{\phi_{23}}$ & $\left(\begin{array}{lll}0 & b & -b\end{array}\right)$ \\
\hline$\nu^{T}$ & $\left(\begin{array}{lll}0 & 0 & \sigma\end{array}\right)$ \\
\hline $\bar{\nu}$ & $\left.\begin{array}{lll}0 & 0 & \sigma\end{array}\right)$ \\
\hline
\end{tabular}

Table 3: Vacuum expectation values. Phases are not shown

\subsection{Quark masses}

The operators of eqs (14-17) with the VEVs of Table 3 generate the Yukawa matrices of the form given in eq(5). This is of the correct form to reproduce the "experimental" form for the mass matrices given in eqs(11) and (2), once the appropriate expansion parameters are inserted as discssed in Section 2.1. Note that the leading operators require $\left|b^{\prime}\right|=\left|c^{\prime}\right|,|b|=|c|,\left|a^{\prime}\right|=1$, $|a|=1$. This follows from the vacuum alignment of the $\phi_{23}, \bar{\phi}_{23}$ fields. However subleading operators will spoil these relations. Keeping all the contributions in eqs(14-18) we obtain the form

$$
\frac{M}{m_{3}}=\left(\begin{array}{ccc}
\epsilon^{8} & \lambda \epsilon^{3}+O\left(\epsilon^{4}\right) & \lambda \epsilon^{3}+O\left(\epsilon^{4}\right) \\
-\lambda \epsilon^{3}+O\left(\epsilon^{4}\right) & \epsilon^{2} & \epsilon^{2}+\lambda^{\prime} \epsilon^{3} \\
-\lambda \epsilon^{3}+O\left(\epsilon^{4}\right) & \epsilon^{2}+\lambda^{\prime} \epsilon^{3} & 1+\epsilon^{2}+2 \lambda^{\prime} \epsilon^{3}
\end{array}\right)
$$

For the case of the down quark mass matrix the expansion parameter is not very small, $\bar{\epsilon} \simeq 0.15$ and so the corrections can be quite large. Using this freedom we see that the $S U(3)$ model is able to reproduce eqs(11) and (2) and thus the quark masses and mixing angles.

\subsection{Charged lepton masses}

With the lepton assignments to $S U(3)$ triplets as in eq(6) , and assuming the same $Z_{2}$ and $R$ charges as for the quarks, the lepton mass matrix will have the same form as the of the quarks, although the expansion parameter may differ if the mass scale associated with the operators is different. However, since the charged leptons get their mass from the same Higgs as generates the down quark masses, if the operators are dominantly generated by mixing to Heavy Higgs fields the expansion parameter for down quarks and charged leptons will be the same. The same will be true if the heavy charged leptons have the same mass scale as the heavy down quarks. Here we assume this is the case and so one should use the expansion parameter $\bar{\epsilon}$ in eq(22) when computing the lepton masses. However it is not consistent to have identical down and charged lepton mass matrices. The relation $m_{b}=m_{\tau}$ is consistent with the measured values after radiative corrections are included, provided this equality applies at a very high scale (the GUT scale?). However to describe the lighter generations it is necessary to have the approximate relations $m_{s} \simeq m_{\mu} / 3$ and $m_{d} \simeq 3 m_{e}$ at the high scale. This can be achieved through the choice of different Yukawa couplings for the charged leptons and down quarks and it may be that this

choice follows from an underlying GUT as suggested by Georgi and Jarlskog [12]. Since we are 
not attempting here to construct the underlying theory valid at a high (GUT) scale, we will not pursue this possibility further but merely note that the choice of lepton Yukawa matrix of the form

$$
Y_{l}=\left(\begin{array}{ccc}
\bar{\epsilon}^{8} & \lambda \bar{\epsilon}^{3} & \lambda \bar{\epsilon}^{3} \\
-\lambda \bar{\epsilon}^{3} & 3 \bar{\epsilon}^{2} & 3 \bar{\epsilon}^{2} \\
-\lambda \bar{\epsilon}^{3} & 3 \bar{\epsilon}^{2} & 1
\end{array}\right)
$$

provides an excellent fit to the lepton masses. The form is that required by the underlying $S U(3)$ symmetry and we have included a factor 3 to achieve the desired relations for the two light generations while keeping the other couplings the same as for the down quarks.

\subsection{Neutrino masses}

We turn now to neutrino masses. As we shall discuss the choice of vacuum alignment given above allows for near bi-maximal mixing even with the universal form for the Dirac masses! Due to the possibility that neutrinos have Majorana masses, the form of the light neutrino mass matrix is expected to differ substantially from that of the quarks and charged leptons. The general form of the effective mass matrix is given by the see-saw form

$$
m_{e f f}=m_{L R} \cdot M_{R R}^{-1} \cdot m_{L R}^{T}
$$

where $M_{R R}$ is the $3 \times 3$ matrix of Majorana masses for the three generations of right-handed neutrinos and $m_{L R}=Y_{\nu} v_{2}$, where $v_{2}$ is the VEV of the second Higgs doublet, and $Y_{\nu}$ is the neutrino Yukawa matrix coupling the left- to right- handed neutrino components. Here we assume there are no $I_{W}=1$ Higgs fields giving rise directly to a Majorana mass for the lefthanded neutrinos.

\subsubsection{The Dirac mass matrix}

Given the neutrino $S U(3)$ assignments of eq(6) we see that the Dirac mass matrix must be of the same form, eq(22) as the charged lepton and quarks. However the expansion parameter may of course be different. Since in this case the neutrinos get their Dirac mass from the same Higgs as generates the up quark masses, if the operators are dominantly generated by mixing to Heavy Higgs fields the expansion parameter for up quarks and neutrinos will be the same. The same will be true if the heavy neutrinos have the same mass scale as the heavy up quarks. We assume this is the case here. As a result the Yukawa matrix is given by

$$
Y_{\nu}=\left(\begin{array}{ccc}
\epsilon^{8} & \lambda_{\nu} \epsilon^{3}+\lambda_{\nu}^{\prime \prime} \epsilon^{4} & \lambda_{\nu} \epsilon^{3}+\lambda_{\nu}^{\prime \prime \prime} \epsilon^{4} \\
-\lambda_{\nu} \epsilon^{3}+\lambda_{\nu}^{\prime \prime} \epsilon^{4} & a_{\nu} \epsilon^{2} & a_{\nu} \epsilon^{2}+\lambda_{\nu}^{\prime} \epsilon^{3} \\
-\lambda_{\nu} \epsilon^{3}+\lambda_{\nu}^{\prime \prime \prime} \epsilon^{4} & a_{\nu} \epsilon^{2}+\lambda_{\nu}^{\prime} \epsilon^{3} & 1
\end{array}\right)
$$

where we have included the couplings of $O(1)$.

\subsubsection{The Majorana mass matrix}

The dominant (heavy right-handed) Majorana mass term comes from

$$
P_{5} \sim \frac{1}{M} \psi_{i}^{c} \nu^{i} \nu^{j} \psi_{j}^{c}
$$


which gives Majorana mass, $M_{3}=\sigma^{2} / M$, to the third family and mass $M_{1}=\epsilon_{\nu}^{4} \sigma^{2} / M$, to the first family. There are further Majorana masses generated higher dimension operators allowed by the symmetries. The leading ones are

$$
P_{6} \sim \frac{1}{M_{3} M^{5}} \psi_{i}^{c} \phi_{23}^{i} \phi_{23}^{j} \psi_{j}^{c} \nu^{k} \overline{\phi_{3, k}} \nu l \overline{\phi_{23, l}}
$$

leading to the final form for the (heavy right-handed) Majorana mass matrix given by

$$
\frac{M_{R R}}{M_{R R, 33}}=\left(\begin{array}{ccc}
\epsilon_{\nu}^{4} & 0 & \epsilon_{\nu}^{2} \\
0 & \epsilon_{\nu}^{3} & \epsilon_{\nu}^{3} \\
\epsilon_{\nu}^{2} & \epsilon_{\nu}^{3} & 1
\end{array}\right)
$$

The result of this is to give (heavy right-handed) Majorana masses, $M_{1}: M_{2}: M_{3}=\epsilon_{\nu}^{4}: \epsilon_{\nu}^{3}$ : 1 where we have allowed for a new expansion parameter $\epsilon_{\nu}$. Note that if $\epsilon_{\nu}<<\epsilon$ the mixing in the light neutrino sector will be dominated by the Dirac mass matrix. In this case the three Majorana mass eigenstates are along the 1,2 and 3 directions in the basis in which $M_{D}$ is written. This alignment proves crucial in allowing for near bi-maximal mixing.

\subsubsection{The effective mass matrix for the light neutrinos}

The see-saw mechanism gives a light effective Majorana matrix via the see-saw formula of eq(24). The resulting Lagrangian giving the light doublet neutrino masses is then

$$
\begin{aligned}
\mathcal{L} \approx & \frac{\left(\epsilon^{8} \nu_{e}+\left(-\lambda_{\nu} \epsilon^{3}+\lambda_{\nu}^{\prime \prime} \epsilon^{4}\right) \nu_{\mu}+\left(-\lambda_{\nu} \epsilon^{3}+\lambda_{\nu}^{\prime \prime \prime} \epsilon^{4}\right) \nu_{\tau}\right)^{2}}{M_{1}} v^{2} \\
& +\frac{\left(\left(a_{\nu} \epsilon^{2}+\lambda_{\nu}^{\prime} \epsilon^{3}\right) \nu_{\tau}+a_{\nu} \epsilon^{2} \nu_{\mu}+\lambda_{\nu} \epsilon^{3} \nu_{e}\right)^{2}}{M_{2}} v^{2} \\
& +\frac{\left(\nu_{\tau}+\left(a_{\nu} \epsilon^{2}+\lambda_{\nu}^{\prime} \epsilon^{3}\right) \nu_{\mu}+\lambda_{\nu} \epsilon^{3} \nu_{e}\right)^{2}}{M_{3}} v^{2}
\end{aligned}
$$

Maximal atmospheric neutrino mixing follows if we have single right-handed neutrino dominance 13. by choosing the parameter $\epsilon_{\nu}$ such that the first right-handed neutrino gives the dominant contribution to the 23 block of the light effective Majorana matrix. The condition for this is $\epsilon_{\nu}<\epsilon^{2}<1$ and is sufficient to guarantee that the contributions from the second term along the 2 direction in eq( $(29)$ is the next most important and determines the mass and mixing of the second heaviest neutrino state. The resulting mass matrix has heaviest state $\nu_{a}=\left(\nu_{\mu}+\nu_{\tau}\right) / \sqrt{2}$ with mass $\propto \epsilon^{6} / \epsilon_{\nu}^{4}$. Up to the correction terms of order $\epsilon$ this is maximally mixed $\left(45^{0}\right)$ due to the vacuum alignment of $\phi_{23}^{T}$.

Turning to the lighter neutrinos the second term in eq(29) provides the dominant contribution if $\epsilon_{\nu}<\epsilon^{2}$. A novel feature of the $S U(3)$ structure is that it leads to the possibility of near maximal mixing in solar neutrino oscillation too! Due to the $S U(3)$ symmetry the leading contribution from this term is $a_{\nu}^{2} \epsilon^{4}\left(\nu_{\mu}+\nu_{\tau}\right)^{2} / M_{2}$ and thus just adds to the $\nu_{a}$ mass. At subleading order it generates a mass $m_{2} \propto \epsilon^{6} / \epsilon_{\nu}^{3}$ to an orthogonal component to $\nu_{a}$ which is a mixture of $\nu_{\mu}-\nu_{\tau}$ and $\nu_{e}$ at the same order in $\epsilon$. As a result there will be large mixing in this sector too. The ratio of neutrino masses is given by

$$
\frac{m_{2}}{m_{3}} \sim \epsilon_{\nu}<\epsilon^{2}
$$


With this ratio our model may be consistent with either the LOW or quasi-vacuum oscillation solutions but not with the LMA solution. With only two right-handed neutrinos effectively contributing, with the first one being dominant, and the second being subdominant, we may use the analytic results of [14] to estimate the remaining mixing angles in this model, which are all expressed in terms of neutrino Yukawa matrix elements in Eq.25. We have already seem that the atmospheric mixing angle $\theta_{13}$ is given by

$$
\tan \theta_{23}=\frac{Y_{21}^{\nu}}{Y_{31}^{\nu}}=1
$$

with small corrections of order $\left|V_{c b}\right|$ from the charged lepton sector. The contribution to the CHOOZ angle from the neutrino sector $\theta_{13}^{\nu}$ is predicted to be vanishingly small

$$
\theta_{13}^{\nu}=\frac{Y_{11}^{\nu}}{\sqrt{Y_{21}^{\nu}+Y_{31}^{\nu 2}}} \sim \epsilon^{5}
$$

Therefore the CHOOZ angle will originate from the charged lepton sector and from eq.(23) we predict a $\mathrm{CHOOZ}$ angle

$$
\theta_{13} \sim\left|V_{u b}\right| \sim(\bar{\epsilon})^{3} \sim 3 \times 10^{-3} .
$$

The solar angle $\theta_{12}$ is predicted to originate mainly from the neutrino sector, with small corrections of order the Cabibbo angle from the charged lepton sector. Neglecting the small CHOOZ angle, and inserting the maximal atmospheric angle, we find

$$
\tan \theta_{12}=\frac{\sqrt{2} Y_{12}^{\nu}}{Y_{22}^{\nu}-Y_{23}^{\nu}} \sim O(1)
$$

where the leading contribution to $Y_{22}^{\nu}-Y_{23}^{\nu}$ cancels due to vacuum alignment, and the subleading $\epsilon^{3}$ term is of the same order as $Y_{12}^{\nu}$ but comes from a different operator with an independent coefficient, so the solar angle is not precisely predicted but is expected to be large.

Note that the large solar mixing angle is due to the equality, in leading order, of the $(2,2)$ and $(2,3)$ matrix elements of $Y^{\nu}$, which followed from the vacuum alignment made possible by the underlying $S U(3)$. This in turn was motivated by the structure of the quark mass matrices. In particular the near equality of the $(2,2)$ and $(2,3)$ Yukawa matrix elements followed from the smallness of $V_{c b}$. The possibility that the $(2,2)$ and $(2,3)$ matrix elements should be equal inleading order has been suggested by the recent data, particularly on the $B_{s}$ lifetime [7].

\subsection{Soft Mass Terms}

One of the main motivations for a non-Abelian family symmetry is the need to solve the flavour problem in supersymmetric models, suppressing large contributions to FCNC coming from virtual diagrams involving non-degenerate squark and slepton masses. Here we consider whether the $S U(3)$ family symmetry is able to achieve this. Generically soft mass terms arise from Dterms of the form $\left(\psi_{i}^{\dagger} \psi_{i} S^{\dagger} S\right)_{D}$ where $S$ is some supersymmetry breaking singlet field which has a non-vanishing F-term $F_{S}$, leading to soft scalar masses $\left|F_{S}\right|^{2}\left(\tilde{\psi}_{i}^{\dagger} \tilde{\psi}_{i}\right) / M^{\prime 2}$. The $S U(3)$ family symmetry therefore ensures that the leading order soft scalar masses are proportional to the unit matrix in family space. However the $S U(3)$ breaking Higgs fields will lead to important corrections to the soft masses. The leading correction is obtained from $\left(\left(\psi_{i} \phi_{3}^{i}\right)\left(\psi_{j} \phi_{3}^{j}\right)^{\dagger} S^{\dagger} S\right)_{D} / M^{\prime 4}$. This 
leads to a contribution to the third family soft scalar masses suppressed by a factor $O\left(a_{3} / M^{\prime}\right)$. The second family receives soft scalar mass corrections from $\left(\left(\psi_{i} \phi_{23}^{i}\right)\left(\psi_{j} \phi_{23}^{j}\right)^{\dagger} S^{\dagger} S\right)_{D} / M^{\prime 4}$ corresponding to a suppression factor of $O\left(b / M^{\prime}\right)$. Here we have allowed for a new mass scale, $M^{\prime}$, associated with these operators.

The detailed dynamical origin of the fermion mass operators discussed above may play a role in determining the magnitude of $M^{\prime}$. For example if the fermion mass operators originate from a Higgs messenger sector, then the higher order corrections to the leading soft operators could be highly suppressed, $M^{\prime}>>M$ because they do not involve the Higgs sector. If they originate from fermion messenger sectors then, since the expansion parameter is larger in the down sector than in the up sector, this may lead to right-handed scalar down mass corrections dominating over right-handed scalar up mass corrections, and so on. Provided $M^{\prime} \geq M^{u}$, the FCNC should be adequately suppressed due to the underlying $S U(3)$ symmetry.

The $S U(3)$ breaking effects can also give important corrections to vacuum alignment. Recall that the equality of the soft mass components $m_{23,2}^{2} \phi_{23,2}^{2}=m_{23,3}^{2} \phi_{23,3}^{2}$ was crucial in obtaining the vacuum $\phi_{23}=(0, b, b)$. The operator

$$
\frac{1}{M^{\prime \prime 4}}\left(\left(\phi_{23, i} \overline{\phi_{3}^{i}}\right)\left(\phi_{23, j} \overline{\phi_{3}^{j}}\right)^{\dagger} S^{\dagger} S\right)_{D}
$$

can give large corrections to the third component soft mass $m_{23,3}$ spoiling the vacuum alignment mechanism. Allowing for this splitting one finds $\phi_{23}=\left(0, b_{2}, b_{3}\right)$ where $b_{2} / b_{3}=m_{23,2}^{2} / m_{23,3}^{2}$. In order for the vacuum alignment to be preserved it is necessary for $a_{3} / M^{\prime \prime}$ to be small. Whether this is the case depends on the details of the heavy messenger sector.

In summary $S U(3)$ offers an elegant mechanism to make the soft quark and lepton mass terms sufficiently close in mass to avoid unacceptably large contributions to FCNC. Whether the SU(3) protection mechanism is sufficiently robust depends on the details of the heavy messenger sector.

\section{Summary and Conclusions}

In this paper we have constructed a model of fermion masses based on an $S U(3)$ family symmetry. The novel feature of this implementation is the use of the underlying $S U(3)$ to align the vacuum expectation values of the fields spontaneously breaking $S U(3)$. This provides a mechanism for explaining maximal mixing in the atmospheric neutrino sector in the case of just three light neutrinos. Our model predicts either the LOW or the quasi-vacuum solar solutions and a CHOOZ angle of order $V_{u b}$. Allowing only for unknown Yukawa couplings of $O(1)$, we were able simultaneously to fit the quark and lepton masses and mixing angles in a model in which all left-handed and charge conjugate right- handed fermion states belonged to $S U(3)$ triplets. Given that the lepton mixing angles are large while the quark mixing angles are small it is remarkable it is possible to achieve such a degree of similarity between quarks and leptons. The reason is due to the structure of the neutrino mass matrix and the see-saw mechanism which provides the mechanism to obtain large lepton mixing angles from the structure responsible for small quark mixing angles. A bonus of the scheme is the protection the $S U(3)$ family symmetry offers against large FCNC.

The model we have constructed is the low energy effective theory coming from Beyond the Standard Model physics. While it is of interest to constuct the underlying theory, it is clear that 
there is considerable flexibility in its structure. For example we can construct a Higgs messenger model in which all the higher dimension operators responsible for the light fermion masses comes from mixing with heavy Higgs states. Alternatively there are examples in which an underlying string theory gives rise to the same effective low energy structure discussed here. We hope to address these questions in a future publication.

\section{Appendix - Vacuum Alignment}

Following Section 3.2 we start with the vacuum structure

$$
<\phi_{3}>=\left(\begin{array}{c}
0 \\
0 \\
a_{3}
\end{array}\right), \quad<\overline{\phi_{2}}>=\left(\begin{array}{c}
0 \\
a_{2} \\
0
\end{array}\right) \text {. }
$$

which is to be triggered by radiative corrections. We assume that $m_{3}^{2}$ becomes negative at a much larger scale than $m_{\overline{2}}^{2}$ so that ultimately we will have $a_{3}^{2} \gg a_{2}^{2}$. For radiativre corrections to trigger a large symmetry breaking scale in a supersymmetric theory the VEVs must develop along D-flat directions. Suppose there are scalar fields transforming as $\overline{3}$ under $S U(3)$ and that $\overline{\phi_{3}}$ has the smallest soft mass amongst these. Then if $m_{3}^{2}\left(\Lambda^{2}\right)+m_{\overline{3}}^{2}\left(\Lambda^{2}\right)<0$ at some scale $\Lambda$ then a VEV for $\phi_{3}, \overline{\phi_{3}}$ will appear and to leading order D-flatness will align it with $<\phi_{3}>$,

$$
<\overline{\phi_{3}}>=\left(\begin{array}{c}
0 \\
0 \\
a_{3}
\end{array}\right) \text {. }
$$

where $a_{3} \simeq \Lambda$.

Consider now the additional fields $\phi_{23}, \overline{\phi_{23}}$. Throughout the analysis the important effect Dterms must be taken into account, since these terms play an important role in determining which VEVs occurs and in vacuum alignment. We assume that $\phi_{23}$ has a positive mass squared and its VEV is triggered by minimising $F_{Y}$ from Eq.20 which leads to $<\phi_{23,2} \phi_{23,3}>=\mu^{4} /\left(a_{2} a_{3}\right) \equiv b^{2}$. The mass term $m_{23}^{2} \phi_{23}^{2}=m_{23}^{2}\left(\phi_{23,1}^{2}+\phi_{23,2}^{2}+\phi_{23,3}^{2}\right)$ is then minimised by equal VEVs in the 2 and 3 directions,

$$
<\phi_{23}>=\left(\begin{array}{l}
0 \\
b \\
b
\end{array}\right) \text {. }
$$

Through the choice of the $\mu$ parameter in Eq.20 we can arrange a hierarchy $a_{3} \gg b \gg a_{2}$. Then $a_{2}$ can be made small enough so as not to affect the mass matrices significantly.

Assuming $m_{\overline{3}}^{2}<m_{23}^{2}<0$, radiative symmetry breaking triggers a VEV for $<\overline{\phi_{23}}>$ and minimising $F_{U}$ from Eq.20 aligns it to be orthogonal to $<\phi_{23}>$,

$$
<\overline{\phi_{23}}>=\left(\begin{array}{r}
0 \\
b^{\prime} \\
-b^{\prime}
\end{array}\right) \text {. }
$$


In order to determine $b^{\prime}$ we must allow for subleading corrections to the D-flatness conditions which align $<\overline{\phi_{3}}>$ with $<\phi_{3}>$ ह ,

$$
<\overline{\phi_{3}}>=\left(\begin{array}{c}
0 \\
y \\
a_{3}+x
\end{array}\right) .
$$

Note that the $F_{Y}$ correction to $\phi_{23} \mathrm{VEV}$ is proportional to $a_{2}$ and hence negligible.

The D-terms coming from the generators $T_{3}=\operatorname{diag}(1,-1,0), T_{8}=\operatorname{diag}(1,0,-1), T_{23}=$ $\delta_{i 2} \delta_{j 3}$, are:

$$
\begin{aligned}
\left|D_{3}\right|^{2} & =\left|-y^{2}+b^{2}-a_{2}^{2}-b^{2}\right|^{2}=0 \\
\left|D_{8}\right|^{2} & =\left|a_{3}^{2}+b^{2}-\left(a_{3}+x\right)^{2}-b^{\prime 2}\right|^{2}=0 \\
\left|D_{23}\right|^{2} & =\left|b^{2}+b^{2}-y\left(a_{3}+x\right)\right|^{2}=0
\end{aligned}
$$

Solving for these we find, to leading order in $b / a_{3}$, that $x=0, y=2 b^{2} / a_{3}, b^{\prime}=b$, which leads to the VEVs in Table 3 .

By a similar analysis one can check that the preferred vacuum has zero VEVs in the first components. Allowing for $<\overline{\phi_{3,1}}>=z,<\phi_{23,1}>=\alpha,<\overline{\phi_{23,1}}>=\bar{\alpha}$, and considering the same D-terms as previously and in addition those associated with the generators $T_{12}=\delta_{i 1} \delta_{j 2}$,

$T_{13}=\delta_{i 1} \delta_{j 3}$, and also considering the soft mass terms associated with the scalars $\overline{\phi_{3}}, \phi_{23}, \overline{\phi_{23}}$, it can be shown that for $m_{23}^{2}>2 m_{\overline{23}}^{2}$ that $\bar{\alpha}=\alpha=z=0$, to leading order.

\section{References}

[1] For a recent review of theories fermion masses, see G.G.Ross, TASI lectures, Boulder, 2000.

[2] For a recent review of the experimental status of neutrino oscillations see S. F. King, hepph/0105261.

[3] For an early discussion of non-abelian family symmetries see P. Ramond, "The Family Group In Grand Unified Theories," in C79-02-25.3 hep-ph/9809459.

[4] R. Barbieri, P. Creminelli and A. Romanino, Nucl. Phys. B 559 (1999) 17 hep-ph/9903460; R. Barbieri, L. J. Hall and A. Romanino, Phys. Lett. B 401 (1997) 47 hep-ph/9702315;

R. Barbieri, L. J. Hall, S. Raby and A. Romanino, Nucl. Phys. B 493 (1997) 3 |hepph/9610449];

T. Blazek, S. Raby and K. Tobe, Phys. Rev. D 62 (2000) 055001 hep-ph/9912482;

Z. Berezhiani and A. Rossi, JHEP 9903 (1999) 002 hep-ph/9811447;

A. Aranda, C. D. Carone and R. F. Lebed, Phys. Rev. D 62 (2000) 016009 hep-ph/0002044;

A. Aranda, C. D. Carone and R. F. Lebed, Phys. Lett. B 474 (2000) 170 [hep-ph/9910392].

\footnotetext{
${ }^{6}$ Note $x, y \ll a_{3}$ should not be confused with the fields $X, Y$ which we have zero VEVs from F-flatness.
} 
[5] The literature is vast, including : J.Chkareuli, JETP Lett.32 (1980)684;

Z. G. Berezhiani and J. L. Chkareuli, JETP Lett. 35 (1982) 612 [Pisma Zh. Eksp. Teor. Fiz. 35 (1982) 494].

Z. G. Berezhiani, Phys. Lett. B 129 (1983) 99;

Z. G. Berezhiani, Phys. Lett. B 150 (1985) 177;

A. A. Anselm and Z. G. Berezhiani, Phys. Lett. B 162 (1985) 349;

Z. Berezhiani, Phys. Lett. B 417 (1998) 287 [hep-ph/9609342];

Z. Berezhiani and A. Rossi, Nucl. Phys. B 594 (2001) 113 [hep-ph/0003084];

M. Soldate, M. H. Reno and C. T. Hill, Phys. Lett. B 179 (1986) 95;

Y. Koide and S. Oneda, Phys. Rev. D 36 (1987) 2867;

R. Kitano and Y. Mimura, Phys. Rev. D 63 (2001) 016008 hep-ph/0008269.

[6] L. Ibanez and G. G. Ross, Phys. Lett. B 332 (1994) 100 hep-ph/9403338;

P. Binetruy and P. Ramond, Phys. Lett. B 350 (1995) 49 [hep-ph/9412385.

[7] R. G. Roberts, A. Romanino, G. G. Ross and L. Velasco-Sevilla, hep-ph/0104088.

[8] P. Ramond, R. G. Roberts and G. G. Ross, Nucl. Phys. B 406 (1993) 19 hep-ph/9303320.

[9] G. Altarelli, F. Feruglio and I Masina, Phys. Lett.B472 (2000) 382.

[10] R. Gatto, G. Sartori and M. Tonin, Phys. Lett. B 28 (1968) 128;

N. Cabibbo and L. Maiani, Phys. Lett. B 28 (1968) 131;

R. J. Oakes, Phys. Lett. B 29 (1969) 683.

[11] C. D. Froggatt and H. B. Nielsen, Nucl. Phys. B 147 (1979) 277.

[12] H. Georgi and C. Jarlskog, Phys. Lett. B 86 (1979) 297.

[13] S. F. King, Phys. Lett. B 439 (1998) 350 hep-ph/9806440;

S. F. King, Nucl. Phys. B 562 (1999) 57 [hep-ph/9904210].

[14] S. F. King, Nucl. Phys. B 576 (2000) 85 [hep-ph/9912492]. 